\title{
Propuesta de arquitectura de microservicios, metodología Scrum para una aplicación móvil de control académico: Caso Escuela Profesional de Obstetricia de la Universidad Nacional Mayor de San Marcos
}

\author{
Microservices architecture proposal, Scrum methodology for a mobile application of \\ academic control: Case of the Universidad Nacional Mayor de San Marcos Professional \\ School of Obstetrics
}

Percy Edwin De la Cruz Vélez de Villa ${ }^{1}$

https://orcid.org/0000-0002-4943-7620

Maycol Henry Espinoza Ramírez ${ }^{2}$

Omar Cuba Estrella ${ }^{3}$

Universidad Nacional Mayor de San Marcos, Perú

Recibido: 14-04-2019

Aceptado: 12-08-2019

\section{Cita recomendada}

De la Cruz, P., Espinoza, M. \& Cuba, O. (2019). Propuesta de arquitectura de microservicios, metodología Scrum para una aplicación móvil de control académico: Caso Escuela Profesional de Obstetricia de la Universidad Nacional Mayor de San Marcos. Hamut'ay, 6(2), 141-158.

http://dx.doi.org/10.21503/hamu.v6i2.1781

\section{RESUMEN}

El objetivo de este documento es presentar una propuesta de un sistema móvil para mejorar la gestión y control académico en la Escuela Profesional de Obstetricia de la Universidad Nacional Mayor de San Marcos. Las fases planteadas del estudio fueron tres. La primera fue de planificación y análisis, relacionadas con la metodología Scrum, donde se visualiza cómo se gestiona el proyecto con la participación de los usuarios. La segunda de diseńo, donde se desarrolló el marco de la arquitectura de microservicios apreciándose su aplicación al buscar unidades funcionales mínimas para su operatividad e independencia. Está integración permitió la realización de la propuesta para el adecuado seguimiento y control a docentes y estudiantes así cómo, para apoyar la gestión académica. El uso del móvil como instrumento de entrada y salida en este proyecto da un cierto nivel de ubicuidad, permitiendo según rol (alumno, docente o

\footnotetext{
${ }^{1}$ Docente Principal, director de la Escuela Profesional de Ingeniería de Sistemas. Licenciado en Computación, Ingeniero de Sistemas, Mg. En Computación e Informática, Estudios doctorales concluidos en Ciencias Administrativas, Ingeniería de Sistemas e Informática. Consultor Informático en TI. Experiencia profesional en empresas del sector Industrial, Pesquero, Metal mecánica. Línea de investigación: Gestión del conocimiento, Big data-Minería de datos, Ingeniería de software. E-mail: pdelacruzv@unmsm.edu.pe

${ }^{2}$ Bachiller en Ingeniería de sistemas, FISI-UNMSM. Ejerce como Ingeniero de Software. Experiencia profesional en proyectos estatales y privados, en la UNMSM (Quipucamayoc), Banco Falabella, Entel, Profuturo AFP, Interbank. Conocimiento de programación Java, servicios web, arquitecturas monolíticas, microservicios, Scrum, RUP, Oracle Suite, Mysql, Spring, Java, GIT, SVN, Jenkins, Docker, AWS Cloud, React Native, Javascript. E-mail: mhersc1@gmail.com

${ }^{3}$ Bachiller en Ingeniería de sistemas, FISI-UNMSM. Se desempeña como analista programador en java. Experiencia profesional en proyectos estatales en la UNMSM (Quipucamayoc), Centro de Producción FISI, RENIEC. Conocimiento de: Programación Java, desarrollo web, arquitecturas monolíticas, microservicios, Scrum, RUP, ORACLE Suite, Mysql, Spring, GIT, SVN. E_mail: cuba.omar13@ gmail.com
} 
director) usar las funcionalidades y lograr esa integración y explotación de la información orientadas al logro de los estándares 14, 20 y 30 exigidos por el Sistema Nacional de Evaluación, Acreditación y Certificación de la Calidad Educativa de Perú.

Palabras clave: calidad educativa, control académico, microservicios, Scrum.

\begin{abstract}
The objective of this document is to present a proposal for a mobile system to improve the management and academic control at the Professional School of Obstetrics of the San Marcos National University. The proposed phases of the study were three. The first one was related to planning and analysis, related to the Scrum methodology, which shows how the project is managed with the participation of the users. The second phase is related to design, where the microservices architecture framework was developed, observing its application in terms of looking for minimum functional units for its operability and independence. This integration allowed the implementation of the proposal for the adequate monitoring and control of teachers and students as well as, to support the academic management. The use of the mobile as an input and output instrument in this project gives a certain level of ubiquity, allowing according to the role (student, teacher or manager) to use the functionalities and achieve that integration and handling of the information oriented to the achievement of standards 14, 20 and 30 required by the National System of Assessment, Accreditation and Certification of Educational Quality of Peru.
\end{abstract}

Keywords: educational quality, academic control, microservices, Scrum.

\section{INTRODUCCIÓN}

Según rankings internacionales de las 100 primeras universidades, ninguna universidad peruana se encuentra ubicada entre las 30 primeras (Quacquarelli, 2011). Otros indicadores estadísticos como COMEXPERU (2011), manifiestan una desigualdad de oportunidades para el acceso a la educación universitaria, producto del centralismo con respecto a las provincias manifestándose una desigualdad de acceso y oportunidades, asimismo, la creación de muchas y diversas universidades privadas que no cumplen con los estándares establecidos para el proceso de licenciamiento de la Superintendencia Nacional de Educación Superior Universitaria (SUNEDU), por lo cual para garantizar un buen nivel competitivo, se exije que primero sean licenciadas por SUNEDU y posteriormente optar por una acreditación. En concor- dancia con la publicación de SINEACE (Yanada, Rivera, \& Castro, 2012), el Perú se encontraba por debajo del rango promedio con lo que respecta a calidad educativa en comparación con otros países, siendo evidente que había problemas en la educación universitaria en Perú. Quacquarelli (2019) manifiesta que del ranking de las 100 mejores universidades latinoamericanas las mejores ubicadas son, en el puesto 21 Pontificia Universidad Católica del Perú, en el puesto 70 Universidad Peruana Cayetano Heredia, y en el puesto 74 Universidad Nacional Mayor de San Marcos. Con la finalidad de revertir estas dificultades es creado el Sistema Nacional de Evaluación, Acreditación y Certificación de la Calidad Educativa (SINEACE) para apoyar la mejora de la calidad universitaria mediante estándares. La Facultad de Medicina de la Universidad Nacional Mayor de San Marcos (UNMSM) desde la Escuela Profesio- 
nal de Obstetricia (EPO) es consciente de que se requiere cumplir con los estándares para continuar posicionándose dentro del ámbito académico, y al tener ciertas falencias que han mermado en una calidad no idónea en algunas de sus áreas, como la información de las clases referidas al registro de notas, la toma de asistencia, la cual se registra en diferentes medios como: papel físico, archivo Excel u otros, no se cuenta con un sistema centralizado, que permita disponer con dicha información, generando retrasos en conseguir y entregar la información entre el profesor y los alumnos. Para superar estos inconvenientes algunos profesores acuden a aplicaciones informáticas (correo, Facebook, WhatsApp, etc.) para comunicar avisos, tales como, el registro de notas, la lista de no asistencia, falta a clase por enfermedad, comunicados de postergación de clase por feriado $\mathrm{u}$ otros acontecimientos, lo cual causa pérdida de tiempo, genera insatisfacción al no tener todos los estudiantes el acceso de esta información para ver sus avances académicos, y en muchos casos que ellos no puedan utilizar en sus hogares esta tecnología, pero, lo más álgido por lo general, es que no se llega a cumplir todos los temarios del sílabo de los cursos en su totalidad. No se puede hacer un seguimiento a todas estas problemáticas, ya que la información impartida en las clases, la ejecución curricular (sílabo), las asistencias de alumnos y profesores, y las notas se formalizan y se registran a fin de ciclo y no está a disposición para su uso y transferencia.

Los aspectos descritos líneas arriba motivaron a que se realice la propuesta del diseño de un sistema distribuido móvil que abarque el control académico y la gestión en la EPO. Se usará la metodología Scrum para la planificación y análisis del proyecto que exige la participación activa de los usuarios en su proceso de desarrollo (Fernández \& Cadelli, 2014), por otro lado, para el diseño nos apoyaremos con la arquitectura de microservicios, que tiene estructura modular, independiente, fáciles de implementar, permitiendo alta cohesión y bajo acoplamiento. revisión de la literatura, para lo cual se seleccionó material bibliográfico y documental de libros digitales relacionado a arquitecturas SOA y microservicios, así como cada uno de los componentes que la conforman, en repositorios como: Scielo, ACM, IEEE, Redalyc, Scopus y WOS.

Para la búsqueda se utilizaron descriptores como: Arquitecturas de software: SOA y microservicios, sistema de Control, Performance Management Control (PMC), Applied Computing, Enterprise Computing, Service-oriented Architecture, Computers and information processing, Distributed computing y distributed information system. Para la selección de búsqueda se tuvo como criterio de inclusión, que los artículos sean de los últimos 5 años.

\section{Sistema Nacional de Evaluación, Acreditación y} Certificación de la Calidad

En el año 2006 se decreta la ley 28740 referida a la creación del Sistema Nacional de Evaluación, Acreditación y Certificación de la Calidad (SINEACE) cuyo objetivo es apoyar a las instituciones educativas a conseguir la acreditación mediante un proceso de evaluación (SINEACE, 2016). En el año 2014, El modelo de acreditación de SINEACE es reorganizado debido a la creación de la Ley universitaria 30220. Este nuevo modelo se basa en una matriz de 34 estándares, de los cuales se han tomado en cuenta tres de ellos, tabla 1, debido a que solo estos están relacionados y pertenecen a la parte académica que corresponde a este estudio.

\section{Tabla 1}

Tres estándares del nuevo modelo SINEACE, según Ley universitaria 30220

\begin{tabular}{ll}
\hline Estándar & \multicolumn{1}{c}{ Descripción } \\
\hline Estándar 14. & El programa de estudios debe evaluar \\
$\begin{array}{l}\text { Selección, evalua- } \\
\text { ción, capacitación y } \\
\text { perfeccionamiento }\end{array}$ & $\begin{array}{l}\text { con el fin de identificar necesidades } \\
\text { para capacitaciones. El perfeccio- } \\
\text { namiento se refiere a actualización, } \\
\text { innovación pedagógica y manejo de } \\
\text { tecnologías de información de los } \\
\text { profesores. }\end{array}$
\end{tabular}

\section{Método}

Para el fundamento de la propuesta se hizo una 


\begin{tabular}{ll}
\hline Estándar & \multicolumn{1}{c}{ Descripción } \\
\hline $\begin{array}{l}\text { Estándar 20. Segui- } \\
\text { miento al desempeño } \\
\text { de los estudiantes. }\end{array}$ & $\begin{array}{l}\text { El programa de estudios debe con- } \\
\text { templar el seguimiento del desempe- } \\
\text { ño de los estudiantes y ofrecer apoyo } \\
\text { como servicios de tutoria o reforza- } \\
\text { miento en cursos. }\end{array}$ \\
$\begin{array}{ll}\text { Estándar 30. Sistema } \\
\text { de información y } \\
\text { comunicación }\end{array}$ & $\begin{array}{l}\text { implementado un sistema de informa- } \\
\text { ción y comunicación que apoye a la } \\
\text { gestión académica y administrativa. }\end{array}$ \\
\hline
\end{tabular}

Fuente: Adaptado de SINEACE (2016).

Según Moromi (2002), la calidad académica universitaria esta engranada con una serie de factores que se tornan necesarios para que esta sea reconocida a nivel académico, profesional y empresarial, siendo uno de ellos la ejecución curricular (control o seguimiento del sílabo o currículo del curso) en el rendimiento académico de los estudiantes universitarios. Asimismo, demuestra la importancia que tiene el cumplimiento del sílabo en la aprobación de una asignatura, y como esta se relaciona directamente entre la ejecución curricular y el rendimiento académico, y, además, resalta la correlación entre la percepción positiva que tienen los alumnos con la ejecución curricular con un mejor rendimiento académico. Otro factor viene a ser la asistencia a clases, según Rodriguez \& Herrera, (2009), la asistencia a clases es un factor determinante para obtener el rendimiento académico esperado. Como conclusión del estudio realizado por estos autores, se demuestra que la asistencia a clases tanto práctica como teórica influye en la superación o mejor rendimiento académico en los estudiantes universitarios. Otro de los aspectos importantes es el desempeño académico del docente con base en los alumnos; según Sifuentes (2018), en su estudio indica que hay una relación significativa en el desempeño académico del docente universitario con la satisfacción de los estudiantes, incide en el rendimiento académico. Para Tolentino (2014) menciona que hay una relación significativa entre el desempeño docente universitario y la satisfacción de los estudiantes del programa.

\section{Sistemas de control de gestión}

Horngren, Datar \& Foster, (2000), definen al sis- tema de control de gestión como "Un sistema que adquiere y usa información que ayuda a la coordinación del planeamiento y las decisiones del control organizacional con el objetivo de mejorar las decisiones colectivas dentro de la organización”. Por su parte Abernethy \& Chua, (1996), indican que es un sistema que combina mecanismos de control diseñados e implementados por los administradores para incrementar la probabilidad de que los líderes de la organización se comporten de una manera consistente con los objetivos de esta. Por lo tanto, un Sistema de control de Gestión debe ser capaz de medir y gestionar el desempeño organizacional, alineados con la generación de valor para la organización, particularmente dirigido a grupos que desempeñen un papel estratégico. La necesidad de controlar el desempeño basado en los flujos de información consistente es la motivación de ser en los sistemas de control de gestión.

La evaluación del desempeño organizacional es trascendental para cualquier tipo de organización, uno de los motivos más importantes por el que las organizaciones deben implementar un sistema de evaluación y control de gestión de sus recursos humanos, es para saber si sus trabajadores están efectivamente contribuyendo al logro de los objetivos institucionales establecidos (Sánchez, 2011), al ser un conjunto de medidas que tratan de estar alineadas con la estrategia de la organización. Además, busca la satisfacción de los stakeholders e influye en todos los actores del negocio a mejorar sus actividades integrando la estrategia, los procesos y los recursos.

Una de las funciones de la evaluación de desempeño es la de proveer información valiosa para la toma de decisiones, siendo este el apoyo principal para el proceso de planeamiento y control, así como mantenerse alineados con los objetivos trazados de la organización. Otra importante funcionalidad es la "señalización", esto quiere decir, mostrar a los empleados la importancia de los aspectos estratégicos establecidos y brindar información no financiera a los stakeholders, tales como la innovación, operaciones, niveles de satisfacción del cliente, entre otros.

Según Che \& Rapiah, (2013) el Sistema de Control de Gestión (CMS) facilita la gestión de los 
entornos crecientes en complejidad. Por otro lado, los Sistemas de Gestión de Rendimiento (PMS) son más horizontales; orientado al proceso y centrado en las necesidades de los grupos de interés. Consecuentemente, es interesante considerar MCS en el diseño de PMS para superar la debilidad del PMS tradicional y mejorar en general el rendimiento.

Para Ferreira \& Otley, (2006) una forma de evaluar a los sistemas de control de gestión es a través del framework "Gestión del desempeño y control", cuya finalidad es capturar la estructura y funcionalidad de los sistemas de control de gestión. A continuación, Tabla 2, se listan los 12 tópicos centrales y las preguntas con las que éstas son formuladas:

Tabla 2

Tópicos centrales y preguntas de sistema de control de gestión

\begin{tabular}{ll}
\hline Tópicos & \multicolumn{1}{c}{ Preguntas } \\
\hline Misión y Visión & ¿Cuál es la visión y misión de la orga- \\
& nización y como se llama la atención \\
& de los gerentes y empleados? ¿Qué \\
& mecanismos, procesos y redes son \\
& usados para transmitir los propósitos \\
& y objetivos generales de la organiza- \\
& ción hacia sus miembros?
\end{tabular}

\begin{tabular}{|c|c|}
\hline Tópicos & Preguntas \\
\hline & $\begin{array}{l}\text { como se encarga de establecer los } \\
\text { objetivos de desempeño apropiados } \\
\text { para ellos y que tan difíciles son esos } \\
\text { objetivos? }\end{array}$ \\
\hline $\begin{array}{l}\text { Evaluación del des- } \\
\text { empeño }\end{array}$ & $\begin{array}{l}\text { ¿Qué procesos, si los hay, sigue } \\
\text { la organización para evaluar el } \\
\text { desempeño individual, grupal y } \\
\text { organizacional? ¿Las evaluaciones } \\
\text { de desempeño son principalmente } \\
\text { objetivas, subjetivas o mixtas y que } \\
\text { tan importantes son la información y } \\
\text { los controles formales e informales en } \\
\text { estos procesos? }\end{array}$ \\
\hline $\begin{array}{l}\text { Sistema de recom- } \\
\text { pensas }\end{array}$ & $\begin{array}{l}\text { ¿Qué procesos, si los hay, sigue } \\
\text { la organización para evaluar el } \\
\text { desempeño individual, grupal y } \\
\text { organizacional? ¿Las evaluaciones } \\
\text { de desempeño son principalmente } \\
\text { objetivas, subjetivas o mixtas y que } \\
\text { tan importantes son la información y } \\
\text { los controles formales e informales en } \\
\text { estos procesos? }\end{array}$ \\
\hline Flujos de información & $\begin{array}{l}\text { ¿Qué flujos específicos de informa- } \\
\text { ción (retroalimentación y avance), } \\
\text { sistemas y redes tiene implementada } \\
\text { la organización para respaldar el } \\
\text { funcionamiento de sus sistemas de } \\
\text { gestión de desempeño? }\end{array}$ \\
\hline $\begin{array}{l}\text { Tipos de uso de los } \\
\text { flujos }\end{array}$ & $\begin{array}{l}\text { ¿Qué tipo de uso se hace de la infor- } \\
\text { mación y de los diversos mecanismos } \\
\text { de control establecidos? ¿Se pueden } \\
\text { caracterizar estos usos en términos } \\
\text { de diversas tipologías en la literatura? } \\
\text { ¿Cómo difieren los controles y sus } \\
\text { usos en diferentes niveles jerárqui- } \\
\text { cos? }\end{array}$ \\
\hline $\begin{array}{l}\text { Cambios en el Siste- } \\
\text { ma PMC }\end{array}$ & $\begin{array}{l}\text { ¿Cómo se han modificado los PMS } \\
\text { a la luz de las dinámicas de cambio } \\
\text { de la organización y su entorno? } \\
\text { ¿Se han realizado los cambios en } \\
\text { el diseño o uso de PMS de manera } \\
\text { proactiva o reactiva? }\end{array}$ \\
\hline $\begin{array}{l}\text { Fuerza y consistencia } \\
\text { de los componentes }\end{array}$ & $\begin{array}{l}\text { ¿Qué tan fuertes y coherentes son los } \\
\text { vínculos entre los componentes de los } \\
\text { PMS y las formas en que se utilizan } \\
\text { (como indica en las } 11 \text { preguntas } \\
\text { anteriores)? }\end{array}$ \\
\hline
\end{tabular}

Fuente: Adaptado de (Ferreira \& Otley, 2006) citado en (Beuren \& Teixeira, 2014).

Aunque existen otros tipos de metodologías como el Proceso Unificado Racional (RUP), éste es una metodología estándar más usada para el análisis, implementación y documentación de sistemas orientados a objetos, de acuerdo a Fernández $\&$ 
Cadelli, 2014. Luego de realizar las comparaciones, en este estudio se eligió aplicar la metodología Scrum.

\section{Scrum}

Según Fernández \& Cadelli, (2014), Scrum es una metodología ágil de gestión de proyectos cuyo objetivo primordial es elevar al máximo la productividad de un equipo. Pone su atención y hace foco sobre valores y prácticas de gestión. Delega completamente al equipo la responsabilidad de decidir la mejor manera de trabajar para ser lo más productivos posibles, es decir, es flexible y los integrantes del equipo pueden optar por organizar la forma de interactuar entre ellos. Toma el cambio como una forma de entregar al final del desarrollo algo más cercano a la verdadera necesidad del cliente. Como metodología ágil está basado en los siguientes principios ágiles:

\section{Tabla 3}

Principios de manifiesto ágil

$$
\text { Principios de Manifiesto Ágil }
$$

Satisfacer al cliente mediante tempranas y continuas entregas de software que aporten valor. Entregar software funcional lo más pronto posible.

Predisposición y respuesta al cambio dado que presentan una ventaja competitiva.

Entregar frecuentemente software que funcione y no planificaciones o documentación de análisis o diseño.

La gente del negocio y los desarrolladores deben trabajar juntos, el proceso de negocio debe ser guiado por el cliente por lo que interactuar con el equipo es frecuente.

El proyecto debe contar con individuos motivados y brindarles apoyo.

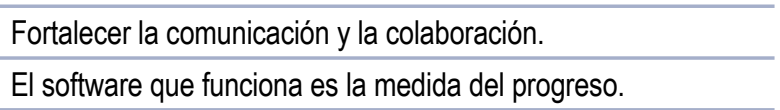

El desarrollo debe ser sostenible por los desarrolladores y usuarios deben colaborar para que esto suceda.

Atención continua en la calidad técnica y buen diseño mejora la agilidad.

La simplicidad es esencial, camino simple consistente con objetivos claros.

El equipo debe ser auto organizado, ellos determinan los objetivos que persiguen.

Reflexión sobre cómo llegar a ser más efectivo en intervalos de tiempo, adaptarse a nuevos entornos.

Fuente: Adaptado de Fernández \& Cadelli, (2014) y Britto, (2016).
El flujo de trabajo de un Scrum, Figura 1, está formado por una serie de elementos que según Fernández \& Cadelli, (2014), estan compuestos por el Product Backlog o lista completa de requerimientos priorizados, que cambia constantemente, no llega a ser totalmente definida; cumple roles, de un Product Owner, que representa a todos los interesados del producto final; además gestiona los requerimientos del Producto Backlog. Posee un Scrum Master, responsable del proceso Scrum, que asegura el desarrollo del proyecto de acuerdo con las prácticas, valores y reglas de Scrum y que progrese según lo previsto. Interactúa con el cliente y el equipo. Encargado de realizar reuniones. En cambio el equipo Scrum es responsable de transformar el Backlog de la iteración en un incremento de la funcionalidad del software (entregable de software).

El Sprint Backlog otro de los elementos hace referencia al trabajo o tareas determinadas por el equipo para realizar en un Sprint. Es decir, la conversión de tareas a un producto funcional. Teniendo como características, que las tareas se estiman en una duración entre 1 a 20 horas de trabajo. Las de mayor duración deben intentar descomponerse en sub-tareas de ese rango de tiempo y que la estimación sea actualizada día a día.

El Sprint, es el período de tiempo durante el que se desarrolla un incremento de funcionalidad; las características del Sprint son: su duración que es de 1 a 4 semanas. Durante el desarrollo del Sprint no se puede modificar el trabajo que se ha acordado en el Backlog, sólo es posible cambiar el curso de un Sprint, abortándolo, y sólo lo puede hacer el Scrum Master si decide que no es viable por alguna de estas razones; la tecnología acordada no funciona, o las circunstancias del negocio han cambiado, o el equipo ha tenido interferencias. El Scrum diario, es una reunión dada cada 24 horas por los integrantes del equipo para determinar el avance de tareas y detectar problemas que impidan no cumplir con las metas del sprint. Para cumplir con lo programado la revisión del Sprint; tiene como fin presentar el producto desarrollado a los usuarios, se analizan y se detectan inconformidades las cuales se pueden planificar y ver en el siguiente sprint. Como último elemento esta la reunión retrospectiva del Sprint, es la reunión del 
equipo Scrum, Product Owner y Scrum Master para ver puntos positivos y negativos del sprint.

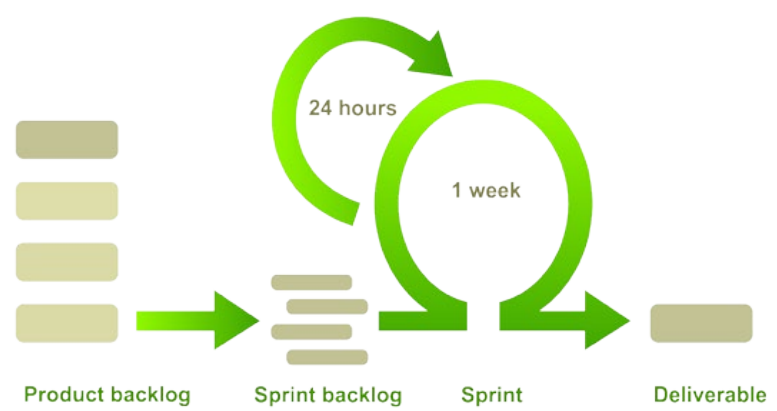

Figura 1.

Flujo de trabajo de SCRUM.

Fuente: Fernández \& Cadelli, (2014)

\section{Arquitectura Orientada a Servicios}

Según Erl, (2016) la Arquitectura Orientada a Servicios (SOA) es un modelo de arquitectura que posiciona a los servicios como un mecanismo esencial para poder incrementar la eficiencia, agilidad y productividad de una empresa. La implementación de SOA puede consistir de la combinación de tecnologías, productos, APIs, extensiones para el soporte de una infraestructura, y otras partes.

Los servicios vienen a ser los componentes unitarios de la arquitectura SOA, son aplicaciones que residen en servidores centralizados, éstos intercambian datos entre sistemas independientemente del lenguaje de programación en el que estén desarrolladas y de la plataforma en dónde se ejecuten. Según Krafzig, Banke, \& Slama, (2004, pp. 58-65), SOA tiene una serie de elementos como:

i. Aplicación Frontend, es uno de los artefactos de SOA que inicia y controla toda la actividad de los sistemas empresariales.

ii. Servicios, componente de software de significado funcional distinto que típicamente encapsula un concepto de negocio de alto nivel y contiene:

A. El contrato del servicio que provee una especificación informal del propósito, funcionalidad, restricciones, y uso del servicio. La forma de esta especificación puede variar, dependiendo del tipo de servicio.

B. La conexión basada en lenguajes como IDL o WSDL. Provee la abstracción e independencia de la tecnología, incluyendo el lenguaje de programación, middleware, protocolo de red y entorno de ejecución. Además, puede imponer detalles semánticos de la funcionalidad y parámetros que no están sujetos a IDL o especificaciones WSDL. La interfaz es expuesta por la conexión del servicio a los clientes que son conectados al servicio usando una red. La implementación de esta interacción denominada "stub service" se usa para desarrollo y pruebas.

C. Implementación del servicio, físicamente provee la lógica del negocio requerido y los datos apropiados. Contiene a artefactos tales como programas, datos de configuración y bases de datos. Compuesto por:

c.1 Lógica de Negocio: es encapsulado por un servicio, es parte de su implementación. Se pone a disposición a través de las interfaces de servicio.

c.2 Data, un servicio que puede también incluir datos.

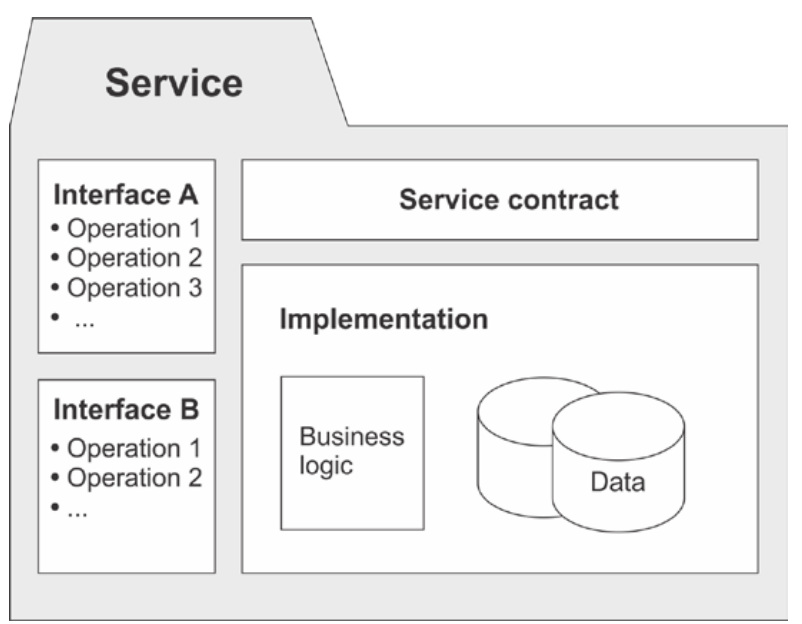

Figura 2.

Componentes de un servicio en SOA.

Fuente: (Krafzig, Banke, \& Slama, 2004)

iii. Servicio de Repositorio: Un repositorio de servicios provee facilidades para descubrir servicios y adquirir toda la información para usar los servicios. 
Propuesta de arquitectura de microservicios, metodología Scrum para una aplicación móvil de control académico: Caso Escuela Profesional de Obstetricia de la Universidad Nacional Mayor de San Marcos

iv. Bus de Servicios: conecta a todos los servicios SOA y aplicaciones frontend.

\section{Arquitectura de Microservicios}

Según Perez-Herrera, (2015) refiere que este tipo de arquitectura tiene como idea principal dividir el software en servicios que sean los más independientes posibles entre ellos distribuidos en diferentes lugares lo cual lo convierte en un sistema distribuido. Según Fowler \& Lewis, (s.f.), los microservicios se definen como un estilo arquitectural en el que múltiples servicios funcionan individualmente desplegados de una manera automatizada, además se comunican con mecanismos ligeros generalmente usando un recurso API basado en HTTP. Según Newman, (2015, pp. 2-3), los microservicios son servicios autónomos que trabajan juntos. Menciona que los aplicativos monolíticos usan un código fuente en muchos casos muy extenso, lo cual dificulta hacer nuevos cambios o el corregir errores sea más difícil, en contraparte a microservicios que persigue. Para esto necesita ser cohesivo, es decir, agrupar código con la misma razón y separar por otro lado código que cumpla diferentes razones. Es por eso que microservicios se enfocan en hacer servicios con base en los límites del negocio, deben ser lo suficientemente pequeños, porque mientras más se reduzca el tamaño más crecen las ventajas. Cada microservicio debe ser desplegado de manera aislada en una plataforma como servicio (PAAS), es por eso que se trata de evitar que múltiples servicios estén en un solo lugar. Las comunicaciones entre estos son mediante llamadas en red. Newman (2015, pp. 45-50), menciona que se puede escoger que principios adoptar en base a la organización, siempre conociendo los riesgos que ello aqueja, en la Tabla 4 se mencionan los principios más resaltantes.

\section{Tabla 4}

Principios de arquitectura de microservicios

\begin{tabular}{ll}
\hline Principios & \multicolumn{1}{c}{ Descripción } \\
\hline Modelar entorno & Que en base a la experiencia de interfaces \\
a conceptos de & $\begin{array}{l}\text { basadas en límites del contexto de negocio } \\
\text { negocios }\end{array}$ \\
& $\begin{array}{l}\text { son más estables. Al modelar en dominios } \\
\text { se asegura que se reflejen los cambios de } \\
\text { los procesos de negocio más fácilmente. }\end{array}$ \\
\hline
\end{tabular}

\begin{tabular}{|c|c|}
\hline Principios & Descripción \\
\hline $\begin{array}{l}\text { Adoptar la cultu- } \\
\text { ra de automati- } \\
\text { zación }\end{array}$ & $\begin{array}{l}\text { Dado que los microservicios aumentan la } \\
\text { complejidad, es bueno contar con fortalecer } \\
\text { una cultura de automatización con herra- } \\
\text { mientas que soporten los microservicios. } \\
\text { El testeo automatizado es esencial, es un } \\
\text { proceso más complejo que los sistemas } \\
\text { monolíticos, también se menciona que se } \\
\text { debe adoptar la integración continua para } \\
\text { una rápida retroalimentación en la calidad } \\
\text { de la producción. }\end{array}$ \\
\hline
\end{tabular}

Esconder deta- Para maximizar la evolución de los microlles internos de servicios independientes, es vital que se implementación esconda los detalles de su implementación y definir bien los límites de negocios es un buen soporte.

Los servicios deben esconder su base de datos para evitar el acoplamiento y para la generación de reportes hacer un movimiento masivo de datos por eventos.

Los servicios deben proveer APIS con tecnología agnóstica (que son servicios que no se preocupan del contexto en el que son llamados) que permite que se usen diferentes tecnologías.

Considerar usar REST que formaliza la separación de la implementación interna y externa.

Descentralizar Para maximizar la autonomía de los microtodas las cosas servicios se necesita buscar constantemente los cambios para delegar el control de los servicios a los equipos.

Asegurar que cada equipo tiene sus servicios es un paso importante, son responsables de los cambios, de cuando lanzan una nueva versión, cada equipo debe ser experto en el dominio del negocio en el cual están creando. Intentar adoptar un modelo de gobierno compartido en los que cada equipo colectivamente comparta responsabilidades de la visión técnica del sistema. La arquitectura, puede evitar enfoques como los buses de servicios empresariales o sistemas de orquestación los cuales centralizan la lógica de negocios y sus servicios son tontos. En vez preferir coreografía sobre orquestación y usar un middleware tonto con endpoint inteligentes que aseguren mantener la lógica asociada y su información dentro de los límites de los servicios ayudando a tener las cosas cohesivas.

Despliegue Se debe tratar de asegurar que los microindependiente servicios puedan ser desplegados por ellos mismos. Aun cuando hay cambios deberían coexistir versiones diferentes que permitan a los clientes cambiar sobre el tiempo. Esto permite optimizar la velocidad de nuev 


\begin{tabular}{|c|c|}
\hline Principios & Descripción \\
\hline & $\begin{array}{l}\text { as características e incrementar la auto- } \\
\text { nomía de los equipos ya que no necesitan } \\
\text { orquestar sus despliegues. } \\
\text { Se puede adoptar por el modelo un servicio } \\
\text { por host que reduce el impacto de afectar } \\
\text { a otro servicio no relacionado. En general } \\
\text { se recomienda no que el despliegue de un } \\
\text { servicio no bloquee a otro y que los clientes } \\
\text { decidan cuando se actualizan ellos mismos }\end{array}$ \\
\hline \multirow[t]{2}{*}{ Aislar las fallas } & $\begin{array}{l}\text { Una arquitectura de microservicios puede } \\
\text { ser más resistente que un sistema monolí- } \\
\text { tico, pero solo si se cuenta con un plan de } \\
\text { fallas dentro del sistema. Si no se cuenta } \\
\text { con el hecho de que puede y va a fallar, el } \\
\text { sistema va a comenzar a fallar y puede ha- } \\
\text { cer al sistema más frágil. Cuando se usan } \\
\text { llamadas no se debe tratar las llamadas } \\
\text { remotas como las llamadas locales en las } \\
\text { que hay una especie de fallo. }\end{array}$ \\
\hline & $\begin{array}{l}\text { Comprender que y como usar los circuit } \\
\text { breakers (interruptores automáticos) para } \\
\text { limitar las consecuencias de un compo- } \\
\text { nente defectuoso. Comprender el impacto } \\
\text { de que pasaría si una parte del sistema se } \\
\text { comporta erróneamente, a veces se debe } \\
\text { sacrificar la disponibilidad o consistencia. }\end{array}$ \\
\hline $\begin{array}{l}\text { Altamente } \\
\text { Observables }\end{array}$ & $\begin{array}{l}\text { No se puede confiar en observar el com- } \\
\text { portamiento de una sola interfaz para ver } \\
\text { si el sistema funciona correctamente, Debe } \\
\text { usarse una vista holística. Usar monitoreo } \\
\text { con transacciones de prueba que simulen } \\
\text { un comportamiento real. Es importante } \\
\text { agregar logs, estadísticas y usar IDS } \\
\text { correlacionados que permitan el rastreo de } \\
\text { las llamadas del sistema. }\end{array}$ \\
\hline
\end{tabular}

Fuente: Adaptado de (Newman, 2015, pp. 45-50).

$\mathrm{Al}$ aplicar la arquitectura de microservicios, es importante tener en cuenta las ventajas que acarrea estos, según Newman, (2015, pp. 4-7).

Tabla 5

Ventajas de la arquitectura de microservicios

\begin{tabular}{ll}
\hline Ventajas & \multicolumn{1}{c}{ Descripción } \\
\hline $\begin{array}{l}\text { Tecnología } \\
\text { Heterogénea }\end{array}$ & $\begin{array}{l}\text { Dado que los microservicios pueden estar } \\
\text { en diferentes sitios independientes unos } \\
\text { de otros se pueden usar diversidades de } \\
\text { tecnología, esto ayuda a encontrar la herra- } \\
\text { mienta apropiada para el tipo de trabajo que } \\
\text { se realice en vez de contar una sola tecno- } \\
\text { logía estandarizada. Con los microservicios } \\
\text { se logra que nuevas tecnologías puedan } \\
\text { ser integradas rápidamente, mayormente } \\
\text { a que los riesgos son mucho menores que } \\
\text { una sola aplicación monolítica. }\end{array}$ \\
\hline
\end{tabular}

\begin{tabular}{|c|c|}
\hline Ventajas & Descripción \\
\hline Resistencia & $\begin{array}{l}\text { La arquitectura de microservicios debe ser } \\
\text { resistente a fallos. Si algo falla y no están } \\
\text { en cascada se puede aislar el problema } \\
\text { y el aplicativo continúa trabajando; por } \\
\text { otro lado, en la arquitectura monolítica } \\
\text { si el servicio falla todo el aplicativo debe } \\
\text { pararse, una forma de mitigar el error se } \\
\text { corría en varias máquinas el aplicativo. Sin } \\
\text { embargo, el autor menciona que se debe } \\
\text { ser cuidadoso para asegurar que los micro- } \\
\text { servicios puedan mejorar la resistencia a } \\
\text { fallos. La red puede y va a fallar para eso la } \\
\text { arquitectura debe estar preparada. }\end{array}$ \\
\hline Escalabilidad & $\begin{array}{l}\text { Cuando se tiene un servicio monolítico } \\
\text { todo debe ser escalado junto, dado que } \\
\text { está relacionado y restringido por lo grande } \\
\text { que es el aplicativo. Con más pequeños } \\
\text { servicios se puede escalar solo los servicios } \\
\text { que se desean }\end{array}$ \\
\hline $\begin{array}{l}\text { Facilidad de } \\
\text { desplegar }\end{array}$ & $\begin{array}{l}\text { En una aplicación monolítica la tarea de } \\
\text { desplegar conlleva muchos riesgos, lanzar } \\
\text { una versión demanda mucho tiempo, ya } \\
\text { que se toma un tiempo para solucionar } \\
\text { todos los posibles errores entre versiones. } \\
\text { Con microservicios se pueden realizar } \\
\text { cambios y desplegar solo dicho servicio, } \\
\text { esto permite nuestro código desplegar } \\
\text { rápidamente, lo cual permite que las nuevas } \\
\text { funcionalidades se puedan ver más rápido }\end{array}$ \\
\hline $\begin{array}{l}\text { Alineamiento } \\
\text { con la organi- } \\
\text { zación }\end{array}$ & $\begin{array}{l}\text { Existen problemas asociados con largos } \\
\text { equipos y largos códigos fuentes. Es } \\
\text { realidad que los equipos más pequeños con } \\
\text { códigos fuentes más pequeños tienden a } \\
\text { ser más productivos. Microservicios ayuda } \\
\text { a alinear la arquitectura a la organización, } \\
\text { ayudando a reducir el número de personas } \\
\text { trabajando sobre un código fuente. }\end{array}$ \\
\hline
\end{tabular}

Fuente: Adaptado de (Newman, 2015; pp. 4-7).

Pero también se debe considerar las desventajas, que según Fowler \& Lewis, (s.f.), se pueden encontrar en el uso de la arquitectura microservicios.

Tabla 6

Desventajas de la arquitectura de microservicios

\begin{tabular}{ll}
\hline Desventajas & \multicolumn{1}{c}{ Descripción } \\
\hline Distribución & La complejidad de realizarse esta arquitec- \\
& tura de forma distribuida es: \\
& 1) Rendimiento: Es realizar llamadas a \\
& funciones remotas, las cuales son lentas a \\
& comparación de llamadas a funciones en el \\
& proceso. Esto se puede mitigar mediante: \\
& Incremeto de granularidad (menos
\end{tabular}


Propuesta de arquitectura de microservicios, metodología Scrum para una aplicación móvil de control académico: Caso Escuela Profesional de Obstetricia de la Universidad Nacional Mayor de San Marcos

\begin{tabular}{|c|c|}
\hline Desventajas & Descripción \\
\hline & $\begin{array}{l}\text { llamadas de funciones remotas asincróni- } \\
\text { camente). } \\
\text { 2) Confiabilidad: Las llamadas a funciones } \\
\text { pueden fallar, si hay más cantidad de micro- } \\
\text { servicios la probabilidad de que ocurra es } \\
\text { más alta. Esto se puede mitigar mediante } \\
\text { la colaboración asincrónica ajustando el } \\
\text { manejo de fallos y pueda resultar en una } \\
\text { mejor recuperación. }\end{array}$ \\
\hline $\begin{array}{l}\text { Consistencia } \\
\text { eventual }\end{array}$ & $\begin{array}{l}\text { Con los microservicios la cantidad de con- } \\
\text { sistencias eventuales va a crecer debido a } \\
\text { que la base de datos esta descentralizada, } \\
\text { mientras que en una aplicación monolítica } \\
\text { todo forma parte de una transacción. Para } \\
\text { esto los desarrolladores deben prever estos } \\
\text { problemas de la consistencia y saber cómo } \\
\text { detectarlos antes de que el código ocasione } \\
\text { estos problemas. }\end{array}$ \\
\hline \multirow[t]{3}{*}{$\begin{array}{l}\text { Complejidad } \\
\text { operacional }\end{array}$} & $\begin{array}{l}\text { Cada unidad es independiente para ser } \\
\text { desplegada; pero esto añade otro tipo de } \\
\text { complejidades como el tener un número }\end{array}$ \\
\hline & $\begin{array}{l}\text { mucho mayor de microservicios que de } \\
\text { aplicaciones. }\end{array}$ \\
\hline & $\begin{array}{l}\text { Manejar esta complejidad requerirá } \\
\text { nuevas habilidades y herramientas como la } \\
\text { entrega continua o la monitorización de los } \\
\text { microservicios; aun así, seguirá siendo más } \\
\text { compleja que una arquitectura monolítica. } \\
\text { Además, se necesita introducir la cultura } \\
\text { "devops" que consiste en una excelente co- } \\
\text { laboración entre desarrolladores, operacio- } \\
\text { nes y todo aquel involucrado en la entrega } \\
\text { del software. }\end{array}$ \\
\hline
\end{tabular}

Fuente: Adaptado de (Fowler \& Lewis (s.f.).

Para decidir que arquitectura es la más idonea en estos casos se debe hacer un análisis de benchmarking, por lo que en la Tabla 7 , se presenta una comparación entre los microservicios y SOA, lo cual permitió en esta investigación decidir utilizar la metodologia descrita.

Tabla 7

Comparativa entre microservicios y SOA

\begin{tabular}{lll}
\hline Asuntos & Microservicios & SOA \\
\hline Despliegue & $\begin{array}{l}\text { Despliegue } \\
\text { individual del } \\
\text { servicio }\end{array}$ & $\begin{array}{l}\text { Despliegue monolítico todos } \\
\text { a la vez }\end{array}$ \\
Equipos & $\begin{array}{l}\text { Cada micro- } \\
\text { servicio es } \\
\text { manejado } \\
\text { por equipos } \\
\text { individuales }\end{array}$ & $\begin{array}{l}\text { Servicios, integración y } \\
\text { la interfaz de usuario son } \\
\text { manejados por equipos } \\
\text { individuales. }\end{array}$ \\
\hline
\end{tabular}

\begin{tabular}{|c|c|c|}
\hline Asuntos & Microservicios & SOA \\
\hline $\begin{array}{l}\text { Interfaz de } \\
\text { usuario }\end{array}$ & $\begin{array}{l}\text { Parte de un } \\
\text { microservicio }\end{array}$ & $\begin{array}{l}\text { Portal de todos los microser- } \\
\text { vicios }\end{array}$ \\
\hline $\begin{array}{l}\text { Alcance de la } \\
\text { arquitectura }\end{array}$ & Un proyecto & Toda la compañía/empresa \\
\hline Flexibilidad & $\begin{array}{l}\text { Despliegue } \\
\text { rápido e inde- } \\
\text { pendiente }\end{array}$ & $\begin{array}{l}\text { Ajusto de procesos de } \\
\text { negocios en el límite de los } \\
\text { servicios }\end{array}$ \\
\hline $\begin{array}{l}\text { Mecanismo } \\
\text { de integra- } \\
\text { ción }\end{array}$ & $\begin{array}{l}\text { Integración } \\
\text { simple y } \\
\text { primitiva }\end{array}$ & $\begin{array}{l}\text { Integración inteligente y } \\
\text { compleja }\end{array}$ \\
\hline Nube nativa & $\mathrm{Si}$ & No \\
\hline $\begin{array}{l}\text { Adminis- } \\
\text { tración / } \\
\text { Gobierno }\end{array}$ & Distribuido & Centralizado \\
\hline $\begin{array}{l}\text { Almacena- } \\
\text { miento de } \\
\text { datos }\end{array}$ & Por unidad & Compartida \\
\hline Escalabilidad & $\begin{array}{l}\text { Mejor } \\
\text { escalabilidad } \\
\text { horizontal. } \\
\text { Elástico }\end{array}$ & $\begin{array}{l}\text { Limitado comparado a } \\
\text { microservicios, cuello de } \\
\text { botella en integrar unidades } \\
\text { o sobrecarga de uso de aná- } \\
\text { lisis de mensajes. Elasticidad } \\
\text { limitada }\end{array}$ \\
\hline Unidad & $\begin{array}{l}\text { Autónomo, } \\
\text { desacopla- } \\
\text { do, propio } \\
\text { contenedor, } \\
\text { escalable } \\
\text { independiente- } \\
\text { mente. }\end{array}$ & $\begin{array}{l}\text { Base de datos compartida, } \\
\text { enlaces de unidades para } \\
\text { servir a procesos de nego- } \\
\text { cios. Bajo acoplamiento }\end{array}$ \\
\hline $\begin{array}{l}\text { Comunica- } \\
\text { ción principal }\end{array}$ & Coreografía & Orquestación \\
\hline Encaja & $\begin{array}{l}\text { Infraestructu- } \\
\text { ras de tamaño } \\
\text { mediano }\end{array}$ & $\begin{array}{l}\text { Infraestructuras de tamaño } \\
\text { largo }\end{array}$ \\
\hline $\begin{array}{l}\text { Tamaño de } \\
\text { servicio }\end{array}$ & $\begin{array}{l}\text { Fina granulari- } \\
\text { dad, pequeño }\end{array}$ & Granularidad fina o gruesa \\
\hline $\begin{array}{l}\text { Versiona- } \\
\text { miento }\end{array}$ & $\begin{array}{l}\text { Debería ser } \\
\text { parte de la } \\
\text { arquitectura, } \\
\text { más abierta a } \\
\text { cambios }\end{array}$ & $\begin{array}{l}\text { Mantenimiento de múltiples } \\
\text { servicios de diferentes } \\
\text { versiones }\end{array}$ \\
\hline $\begin{array}{l}\text { Nivel de Ad- } \\
\text { ministración }\end{array}$ & Anarquía & Centralizado \\
\hline $\begin{array}{l}\text { Localización } \\
\text { de las reglas } \\
\text { de negocio }\end{array}$ & $\begin{array}{l}\text { Servicio } \\
\text { particular }\end{array}$ & Componente de integración \\
\hline
\end{tabular}

Fuente: Cerny, Donahoo, \& Pechanec, (2017).

Con base en las comparaciones mostradas se puede resaltar en términos generales que en este estudio se eligió la arquitectura de microservicios dado que se ajusta más a las necesidades del clien- 
te, debido a que el software se desplegará en una empresa de nivel intermedio como es la EPO, aplicando el escalamiento horizontal, ya que es independiente no requiere de integraciones complejas como buses, tiene componentes con bajo acoplamiento, es flexible por contar con despliegue rápido que no afecta para el despliegue de otros servicios, el gobierno descentralizado de cada servicio contiene la lógica de negocio y su acceso a la información está aislado de otros servicios. Por último, su adaptación es más rápida para los cambios solicitados por los clientes.

\section{Desarrollo de la propuesta}

El alcance en este artículo cubrirá el análisis, planificación y diseño.

\section{Análisis y Planificación.}

Según las características del escenario (Britto, 2016), se optó por la metodología Scrum. Siguiendo sus pasos se conformó el equipo para el desarrollo de la propuesta descrita, Figura 3.

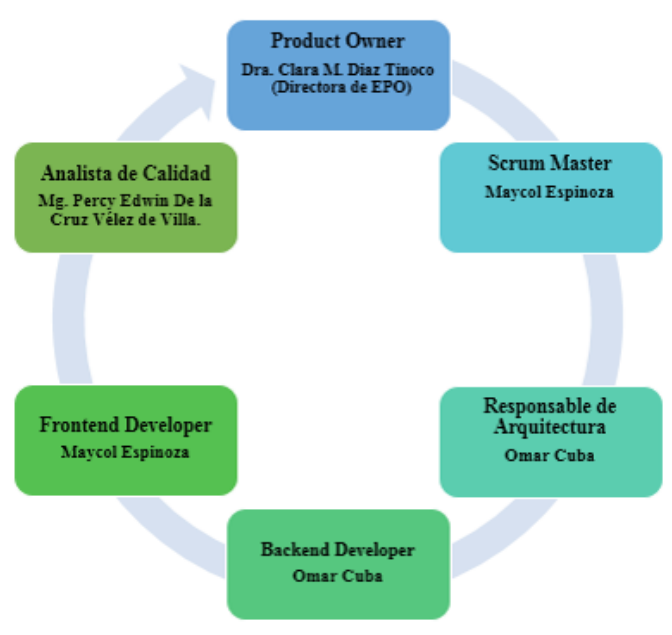

Figura 3.

Equipo Scrum del estudio (2019).

Planificación de los sprints (Inceptions o sprint 0)

En esta etapa participan el Product Owner, Scrun Master e incluimos al responsable de la Arquitec- tura. Generándose el Backlog a partir de las épicas que el proyecto necesitaba. Se identifican las historias de usuario, y el Product Owner prioriza e identifica los hitos a controlar y la estimación del tiempo del proyecto. Se tiene proyectado a 6 sprints (0-5), cada sprint tendrá una duración de un mes. Es decir, la duración estimada del proyecto es de 6 meses. Además, se especifica la arquitectura de proyecto, la figura 4 detalla la misma.

En la Tabla 8, se ha integrado el Backlog general con una estimación referencial.

\section{Tabla 8}

BACKLOG

\begin{tabular}{|c|c|c|}
\hline $\begin{array}{l}\text { BAC- } \\
\text { KLOG }\end{array}$ & Historias de Usuario & $\begin{array}{l}\text { Esti- } \\
\text { ma- } \\
\text { ción }\end{array}$ \\
\hline HU01 & $\begin{array}{l}\text { Como usuario, debo poder autenticarme } \\
\text { en el sistema de manera segura, así } \\
\text { como garantizar el cierre de sesión en mi } \\
\text { dispositivo móvil. }\end{array}$ & 5 \\
\hline HU02 & $\begin{array}{l}\text { Como usuario, debo tener acceso a mis } \\
\text { funcionalidades básicas correspondientes. }\end{array}$ & 3 \\
\hline HU03 & $\begin{array}{l}\text { Como usuario, debo poder visualizar la } \\
\text { relación de cursos, así como la estructura } \\
\text { curricular de cada una de ellas. }\end{array}$ & 4 \\
\hline HU04 & $\begin{array}{l}\text { Como usuario debo poder distinguir los } \\
\text { estados de las clases correspondientes a } \\
\text { un sílabo determinado. }\end{array}$ & 3 \\
\hline HU05 & $\begin{array}{l}\text { Como docente debo ser capaz de iniciar } \\
\text { y finalizar la clase, por consecuente, esta- } \\
\text { blecer su estado como iniciado, finalizado } \\
\text { respectivamente. }\end{array}$ & 5 \\
\hline HU06 & $\begin{array}{l}\text { Como usuario deseo ser capaz de visuali- } \\
\text { zar el detalle de la asistencia de una clase } \\
\text { dada. }\end{array}$ & 5 \\
\hline HU07 & $\begin{array}{l}\text { Como docente, debo poder consultar } \\
\text { las calificaciones de todos mis alumnos } \\
\text { inscritos en una determinada agrupación } \\
\text { curricular }\end{array}$ & 3 \\
\hline HU08 & $\begin{array}{l}\text { Como alumno, debo poder consultar mis } \\
\text { calificaciones relacionadas a una agrupa- } \\
\text { ción curricular determinada. }\end{array}$ & 3 \\
\hline HU09 & $\begin{array}{l}\text { Como docente, debo poder registrar o } \\
\text { modificar una o más de las calificaciones } \\
\text { de los alumnos inscritos en una agrupación } \\
\text { curricular determinada }\end{array}$ & 5 \\
\hline HU10 & $\begin{array}{l}\text { Como usuario, debo ser capaz de visuali- } \\
\text { zar el listado de anuncios realizados por el } \\
\text { docente a lo largo del semestre académico. }\end{array}$ & 3 \\
\hline HU11 & $\begin{array}{l}\text { Como docente, debo poder registrar un } \\
\text { anuncio correspondiente a una agrupación } \\
\text { curricular determinada. }\end{array}$ & 3 \\
\hline
\end{tabular}




\begin{tabular}{|c|c|c|}
\hline $\begin{array}{l}\text { BAC- } \\
\text { KLOG }\end{array}$ & Historias de Usuario & $\begin{array}{l}\text { Esti- } \\
\text { ma- } \\
\text { ción }\end{array}$ \\
\hline HU12 & $\begin{array}{l}\text { Como usuario debo poder ver todas las } \\
\text { preguntas y respuestas relacionadas a una } \\
\text { clase determinada. }\end{array}$ & 5 \\
\hline HU13 & $\begin{array}{l}\text { Como usuario, debo ser capaz de realizar } \\
\text { preguntas y/o responder otras preguntas. }\end{array}$ & 5 \\
\hline HU14 & $\begin{array}{l}\text { Como usuario, debo poder visualizar la } \\
\text { formula correspondiente a una agrupación } \\
\text { curricular. }\end{array}$ & 5 \\
\hline HU15 & $\begin{array}{l}\text { Como docente, debo ser capaz de modifi- } \\
\text { car una formula previamente definida co- } \\
\text { rrespondiente a una agrupación curricular. }\end{array}$ & 3 \\
\hline HU16 & $\begin{array}{l}\text { Como alumno, debo valorizar la clase } \\
\text { impartida por el docente. }\end{array}$ & 3 \\
\hline HU17 & $\begin{array}{l}\text { Como usuario, debo ser capaz de ser } \\
\text { notificado cuando ocurra algún evento } \\
\text { relevante. }\end{array}$ & 5 \\
\hline HU18 & $\begin{array}{l}\text { Como director de escuela debo ser capaz } \\
\text { de ver los reportes de asistencias }\end{array}$ & 5 \\
\hline HU19 & $\begin{array}{l}\text { Como director de escuela debo ser capaz } \\
\text { de ver el reporte de desempeño académico } \\
\text { estudiantil }\end{array}$ & 5 \\
\hline HU2O & $\begin{array}{l}\text { Como director de escuela debo ser capaz } \\
\text { de ver el reporte de valoraciones obtenidas } \\
\text { en las encuestas }\end{array}$ & 5 \\
\hline $\begin{array}{l}\text { SPI- } \\
\text { KE01 }\end{array}$ & Diseño de la arquitectura Backend & 8 \\
\hline $\begin{array}{l}\text { SPI- } \\
\text { KE02 }\end{array}$ & Diseño de la arquitectura Frontend & 8 \\
\hline $\begin{array}{l}\text { SPI- } \\
\text { KE03 }\end{array}$ & $\begin{array}{l}\text { Abordar la consistencia eventual en la } \\
\text { arquitectura de microservicios }\end{array}$ & 5 \\
\hline $\begin{array}{l}\text { SPI- } \\
\text { KE04 }\end{array}$ & $\begin{array}{l}\text { Implementación de Websockets en React } \\
\text { Native }\end{array}$ & 5 \\
\hline
\end{tabular}

Fuente: Cerny, Donahoo, \& Pechanec, (2017).

\section{Definición de sprint}

En los sprints del 1 al 5, la duración aproximada es de 1 mes por sprint y la jornada de trabajo es de lunes a viernes de 9am a 5pm (considerar hora refrigerio). El primer día del sprint comienza con una reunión de planificación de 4 a 7 horas. Aquí se estima la complejidad de la historia para entregar, asimismo se realiza el desglose de las tareas por persona y se asigna un responsable de la historia respectiva. Los siguientes días se realiza una reunión diaria de 15 minutos con el fin de revisar el avance, dificultades que tuviera el equipo. Posteriormente se realizará las tareas pactadas en la planificación. En la tercera semana hay una reunión de refinamiento donde se presenta las historias que vendrán en el siguiente sprint (Se analizan riesgos y problemas etc.). Al final del sprint se realiza la revisión con la dueña del producto en un tiempo estimado de 3 horas (presentar una demostración).

El último día del sprint se realiza la reunión de retrospectiva del equipo, con una duración de 3 horas (para una mejorar continua del equipo).

Se describen en las Tablas 9, 10 y 11, algunos de los sprints de la propuesta que contemplan mayor valor para el usuario final (Control de asistencias $y$ reportes).
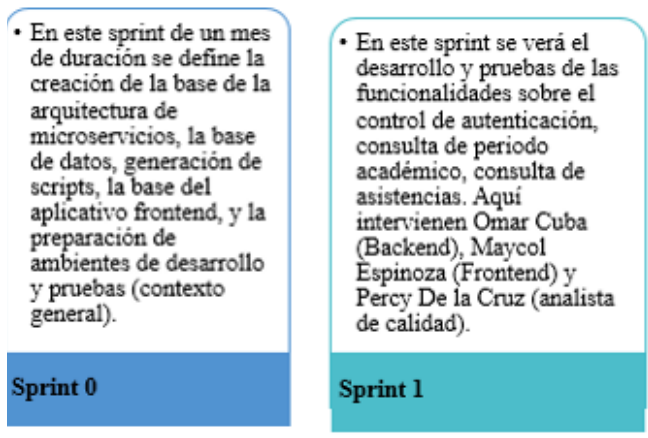

- En este sprint se verá el desarrollo y pruebas del registro de notas, registro de fórmulas generales o especificas $y$ consulta de notas. Aqui intervienen Omar Cuba (Backend), Maycol Espinoza (Frontend) y Percy De la Cruz (analista de calidad)

Sprint 3

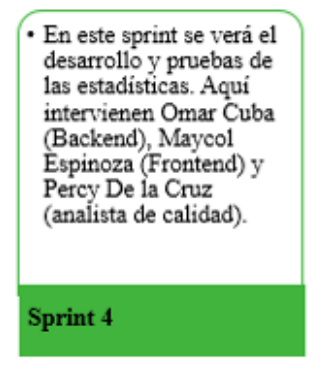

Figura 4.

Descripción del Sprint 0 - 5. Elaboracion propia (2019).

Tabla 9

Sprint 1

\begin{tabular}{|c|c|c|}
\hline $\begin{array}{l}\text { HU / } \\
\text { Spike }\end{array}$ & Tareas & Responsable \\
\hline \multirow[t]{2}{*}{ HUO4 } & $\begin{array}{l}\text { Creación del endpoint, Listar } \\
\text { asistencia clases por agrupación } \\
\text { curricular (MS ASISTENCIA) }\end{array}$ & Omar Cuba \\
\hline & $\begin{array}{l}\text { Creación del endpoint composer } \\
\text { Listar Clases del presente día con } \\
\text { sus respectivos estados (MS Perio- } \\
\text { do Académico + MS Asistencia) }\end{array}$ & Omar Cuba \\
\hline
\end{tabular}




\begin{tabular}{lll}
\hline HU I & Tareas & Responsable \\
\hline & $\begin{array}{l}\text { Desarrollo del semáforo (estado de } \\
\text { la clase) en la GUI Listar Clases del } \\
\text { presente día }\end{array}$ & Maycol \\
\cline { 2 - 2 } & $\begin{array}{l}\text { Despinoza } \\
\text { la clase) en la GUI Listar Clases } \\
\text { por fecha especifica }\end{array}$ & Espinoza \\
\hline $\begin{array}{l}\text { Creación del esquema, tablas, } \\
\text { constraints, queries correspondien- }\end{array}$ & Maycol \\
$\begin{array}{l}\text { tes a la base de datos de ASIS- } \\
\text { TENCIA. }\end{array}$ & \\
\hline $\begin{array}{l}\text { Creación y ejecución de los casos } \\
\text { de pruebas alineados a los criterios } \\
\text { de aceptación establecidos }\end{array}$ & Percy De la \\
\hline
\end{tabular}

Fuente: Elaboración propia, (2019).

\section{Tabla 10}

Sprint 2

\begin{tabular}{|c|c|c|}
\hline $\begin{array}{l}\text { HU / } \\
\text { Spike }\end{array}$ & Tareas & Responsable \\
\hline \multirow[t]{8}{*}{ HU05 } & $\begin{array}{l}\text { Creación del endpoint transaccional } \\
\text { Iniciar Asistencia (MS ASISTEN- } \\
\text { CIA) }\end{array}$ & Omar Cuba \\
\hline & $\begin{array}{l}\text { Creación del endpoint transaccional } \\
\text { Finalizar Asistencia (MS ASISTEN- } \\
\text { CIA) }\end{array}$ & Omar Cuba \\
\hline & $\begin{array}{l}\text { Creación del endpoint transac- } \\
\text { cional Registrar Asistencias (MS } \\
\text { ASISTENCIA) }\end{array}$ & Omar Cuba \\
\hline & $\begin{array}{l}\text { Creación del endpoint Listar Alum- } \\
\text { nos por agrupación curricular (MS } \\
\text { PERIODO ACADEMICO) }\end{array}$ & Omar Cuba \\
\hline & $\begin{array}{l}\text { Integración de los endpoints men- } \\
\text { cionados anteriormente dentro de } \\
\text { la capa Frontend. }\end{array}$ & $\begin{array}{l}\text { Maycol } \\
\text { Espinoza }\end{array}$ \\
\hline & $\begin{array}{l}\text { Implementación del registro de } \\
\text { asistencias de los alumnos en la } \\
\text { GUI Asistencia. }\end{array}$ & $\begin{array}{l}\text { Maycol } \\
\text { Espinoza }\end{array}$ \\
\hline & $\begin{array}{l}\text { Implementación del inicio y } \\
\text { finalización de la clase en la GUI } \\
\text { Asistencia }\end{array}$ & $\begin{array}{l}\text { Maycol } \\
\text { Espinoza }\end{array}$ \\
\hline & $\begin{array}{l}\text { Creación y ejecución de los casos } \\
\text { de pruebas alineados a los criterios } \\
\text { de aceptación establecidos. }\end{array}$ & $\begin{array}{l}\text { Percy De la } \\
\text { Cruz }\end{array}$ \\
\hline \multirow[t]{2}{*}{ HU06 } & $\begin{array}{l}\text { Adaptación del componente acor- } \\
\text { deón en el GUI Asistencias para } \\
\text { añadir el detalle de asistencias }\end{array}$ & $\begin{array}{l}\text { Maycol } \\
\text { Espinoza }\end{array}$ \\
\hline & $\begin{array}{l}\text { Asegurar el comportamiento } \\
\text { responsivo para ambas partes } \\
\text { del acordeón (Detalle de clase y } \\
\text { Listado de asistencias) }\end{array}$ & $\begin{array}{l}\text { Maycol } \\
\text { Espinoza }\end{array}$ \\
\hline
\end{tabular}

\begin{tabular}{lll}
\hline HU I & Tareas & Responsable \\
\hline & $\begin{array}{l}\text { Implementar las "go backs" (re- } \\
\text { gresar atrás Android) en todas las } \\
\text { vistas realizadas hasta el momento. }\end{array}$ & Espinoza \\
\cline { 2 - 3 } & $\begin{array}{l}\text { Creación y ejecución de los casos } \\
\text { de pruebas alineados a los criterios } \\
\text { de aceptación establecidos }\end{array}$ & $\begin{array}{l}\text { Percy De la } \\
\end{array}$ \\
\hline
\end{tabular}

Fuente: Elaboración propia, (2019).

Tabla 11

Sprint 4

\begin{tabular}{|c|c|c|}
\hline $\begin{array}{l}\text { HU / } \\
\text { Spike }\end{array}$ & Tareas & Responsable \\
\hline HU18 & $\begin{array}{l}\text { Creación del endpoint "obtenerEs- } \\
\text { tadisticasAsistencias" (MS ASIS- } \\
\text { TENCIA), servicio encargado de } \\
\text { obtener los porcentaies de faltas }\end{array}$ & Omar Cuba \\
\hline
\end{tabular}
obtener los porcentajes de faltas, tardanzas, asistencias.

Creación de la GUI "Ver reportes Maycol de asistencias", con sus respec- Espinoza tivos filtros: "curso", "agrupación curricular", "docente" y "alumno"

$\begin{array}{ll}\text { Creación y ejecución de los casos } & \text { Percy De la } \\ \text { de pruebas alineados a los criterios } & \text { Cruz } \\ \text { de aceptación }\end{array}$
de pruebas alineados a los criterios Cruz de aceptación establecidos

HU19 Creación del endpoint "obtene- Omar Cuba
rEstadisticasNotas" (MS NOTAS), servicio encargado de obtener los porcentajes según rango de notas. Del mismo modo, con los filtros: "curso", "agrupación curricular", "estudiante".

\begin{tabular}{ll}
\hline Creación del GUI "Ver reportes de & Maycol \\
Desempeño Académico", con sus & Espinoza \\
respectivos filtros; "curso", "agrupa- &
\end{tabular}
respectivos filtros; "curso", "agrupación curricular", "estudiante".

\begin{tabular}{ll}
\hline Creación y ejecución de los casos & Percy De la \\
de pruebas alineados a los criterios & Cruz \\
de aceptación establecidos &
\end{tabular}

H20 Creación del endpoint "obtene- Omar Cuba rEstadisticasEncuestas", servicio encargado de obtener el promedio de valoraciones respecto a un curso y/o agrupación curricular seleccionados.

\begin{tabular}{ll}
\hline Creación del GUI "Ver reporte de & Maycol \\
encuestas", con sus respectivos & Espinoza \\
filtros: "curso" y "agrupación & \\
curricular" & \\
\hline $\begin{array}{l}\text { Creación y ejecución de los casos } \\
\text { de pruebas alineados a los criterios }\end{array}$ & Pruz De la \\
de aceptación establecidos &
\end{tabular}


Propuesta de arquitectura de microservicios, metodología Scrum para una aplicación móvil de control académico: Caso Escuela Profesional de Obstetricia de la Universidad Nacional Mayor de San Marcos

\begin{tabular}{lll}
\hline HU I & Tareas & Responsable \\
Spike & & \\
\hline & $\begin{array}{l}\text { Creación del GUI "Ver comparativo } \\
\text { Notas vs Encuestas", con sus } \\
\text { respectivos filtros "Curso" y "agru- } \\
\text { pación curricular". }\end{array}$ & Maycol \\
\cline { 2 - 2 } & $\begin{array}{l}\text { Creación y ejecución de los casos } \\
\text { de pruebas alineados a los criterios } \\
\text { de aceptación establecidos }\end{array}$ & Percy De la \\
\hline
\end{tabular}

Fuente: Elaboración propia, (2019).

\section{Diseño}

En la figura 5, se describe el diseño de la arquitectura del sistema que soportará la futura implementación del proyecto. Se considera su composición de la siguiente forma: Por el lado cliente (frontend) Eisenman (2016) afirma que usar el framework react native permite la renderización en aplicativos con plataformas móviles como ANDROID e IOS. Por el lado del servidor (backend) se cuentan con los componentes no funcionales que apoyan a nuestra arquitectura de microservicios (config, eureka, gateway y composición), y los componentes funciones, los cuales son los microservicios de autenticación, asistencias, notas, coparticipación, encuesta, notificación, reportes que serán desarrollados con spring boot y cada uno contará con un acceso privado a su base de datos. Para agregar seguridad de acceso a los servicios se hará uso de oauth2 y por último para gestionar los eventos (mediante mensajería) se usará rabbitmq.

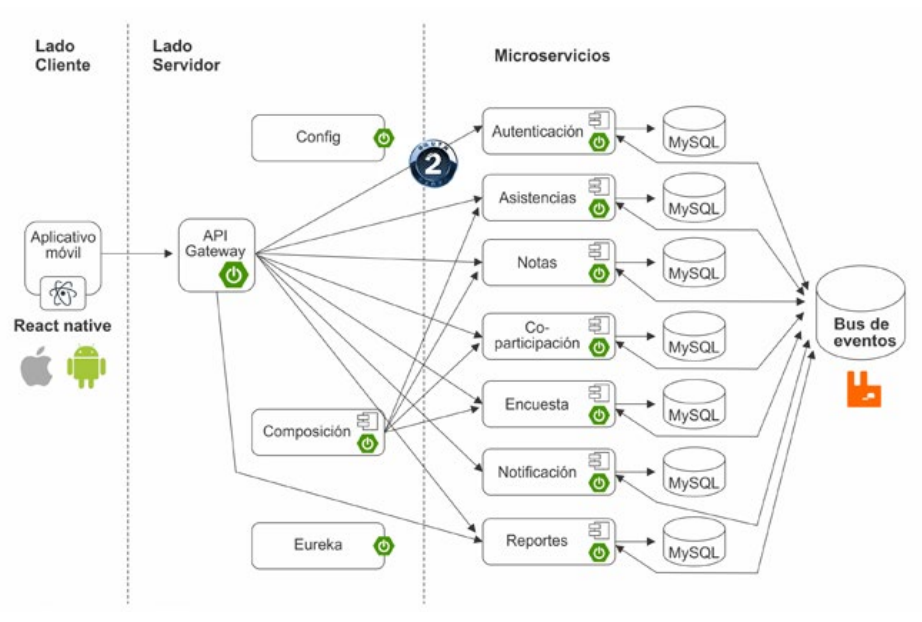

Figura 5.

Diagrama del diseño de la arquitectura del sistema.

Fuente: Elaboración propia (2019).
A continuación, se detallará cada uno de los componentes funcionales en la Tabla 12 y los componentes no funcionales en la Tabla 13 de la arquitectura de microservicios propuesta.

\section{Tabla 12}

Descripción de los componentes no funcionales

\begin{tabular}{ll}
\hline Componentes no funcionales & \multicolumn{1}{c}{ Elementos no funcionales } \\
\hline No contienen lógica de & Eureka-Server. Este servicio \\
negocio, pero aportan en el & usa el componente spring \\
funcionamiento de la arqui- & cloud eureka nos permite \\
tectura de microservicios & realizar el auto-registro, descu- \\
se hace uso del framework & brimiento dinámico y balanceo \\
spring cloud y también se & de carga. (RV, 2016) \\
considera aquí al servicio & \\
composición el cual requiere & \\
el uso de la combinación de & \\
varios microservicios para & \\
las consultas del lado cliente & \\
(frontend). &
\end{tabular}

Config-Server. Este servicio usa el componente spring cloud config el cual usamos la configuración externa de las propiedades de configuración de los microservicios.

Gateway. Este servicio se sitúa entre el cliente y el servidor, transforma la data según lo requerido por el cliente (servicios UI). Actúa como un proxy para el backend, exponiendo un conjunto de APIs especificas al cliente. Haremos uso de zuul, que usa el proxy reverso que funciona como una plataforma compartida para varios microservicios.

Composición. Este servicio usa el patrón API composition, este patrón implementa una consulta(query) al invocar a los servicios que tienen la data por medio de sus APIS y las combina los resultados en un resultado. No cuenta con base de datos (BD).

Fuente: Elaboración propia (2019). 
Tabla 13

Descripción de los componentes funcionales

\begin{tabular}{l} 
Componentes funcionales \\
\hline Aquí se encuentra la lógica \\
de negocio, aquí están los \\
microservicios. Cada uno \\
es independiente entre sí \\
definidos así por límites \\
de negocio, manejan su \\
propia base de datos. Para \\
la seguridad se hace uso de \\
Oauth2 que permite acceso a \\
los servicios cuando el usua- \\
rio se autentica mediante \\
tokens. Por último, se hace \\
uso de rabbit MQ para que \\
los microservicios al registrar \\
cambios se comuniquen \\
entre sí mediante eventos \\
(mensajes). Esto permite la \\
programación asincrónica y \\
el uso de colas en cambio \\
la comunicación mediante \\
HTTP por defecto no es \\
secuencial es decir aumenta \\
el tiempo de ejecución del \\
servicio y no es recomenda- \\
ble a menos que se maneje \\
de forma asincrónica.
\end{tabular}
de forma asincrónica.

\section{Elementos funcionales}

Autenticación. Este microservicio contiene la información relacionada al usuario, roles y perfiles; permite a un usuario con perfiles como (profesor, alumno, directora de escuela) la autenticación en el sistema mediante el ingreso de su usuario y contraseña; también permite realizar el cierre de sesión de un usuario dado. Aquí se usa Oauth2 el cual brinda token al cliente que permitirá el acceso a los microservicios. Cuenta con una BD mysql.

Asistencia. Permite registrar/ actualizar y finalizar las asistencias de las clases, Cuenta con una BD mysql.

\section{Encuesta. Permite realizar} las encuestas con calificaciones serán de 1 a 5 estrellas. Permite obtener la última plantilla de encuesta en estado activo con la cual se realizarán las encuestas. Por último, permite calcular el promedio de encuestas realizadas en base a las clases. Cuenta con una BD mysql.

Coparticipación. Entre las principales funciones de este microservicio serán de "registrar pregunta", referentes a un tema en específico o en general para que puedan ser respondidas por el docente 0 en todo caso por un compañero de clase. "responder respuesta" es el caso consecuente de la acción "registrar pregunta", puede ser realizado por profesores y/o alumnos con el fin de solventar la duda. "Registrar anuncios (por parte de los profesores)", que permite al profesor hacer anuncios de algunos imprevistos 0 avisos que quiera comunicar. Cuenta con BD mysql.

Notas. Los docentes asignados a los grupos de un curso podrán ingresar las notas de las diferentes evaluaciones. etc.

\begin{tabular}{ll}
\hline Componentes funcionales & \multicolumn{1}{c}{ Elementos funcionales } \\
\hline Así como la consulta y el \\
registro de fórmulas generales \\
y específicas. Cuenta con una \\
BD mysql. \\
\hline Periodo Académico. Contiene \\
la información de los cursos \\
en un semestre, tiene la \\
información de los alumnos y \\
profesores, los grupos defini- \\
dos en el curso, la relación de \\
alumnos inscritos en dichos \\
grupos y también la relación de \\
los horarios de las clases para \\
cada grupo. Cuenta con una \\
BD mysql. \\
\hline Notificación \\
Es útil cuando hay cambios o \\
actualizaciones por parte de \\
los alumnos y profesores \\
que es de importancia para \\
los demás, se resaltan las \\
notificaciones de asistencias, \\
notas, anuncios, preguntas y \\
respuestas. Cuenta con BD \\
mysql. \\
\hline Reportes. Permitirá generar re- \\
portes y/o estadísticas. Cuenta \\
con una BD propia para una \\
consulta más rápida, la cual es \\
llenada mediante eventos por \\
mensajería usando rabbitMQ \\
que usan los microservicios de \\
asistencias, notas, encuestas y \\
periodo académico cuando ac- \\
tualizan su propia BD. Cuenta \\
con una BD mysql. \\
\hline
\end{tabular}

Fuente: Elaboración propia (2019).

Perfiles. La propuesta desarrollada para la EPO, tiene 3 perfiles: alumno, profesor y de la directora de escuela. Las interfaces funcionales del menú de la directora de escuela, se aprecia en la figura 6.

Estadísticas: Le permite generar estadísticas en base a las asistencias, notas y encuestas de alumnos y profesores.

Cambiar Rol: Le permite cambiar de rol si tuviera más de uno.

Cerrar Sesión: Le permite cerrar la sesión 


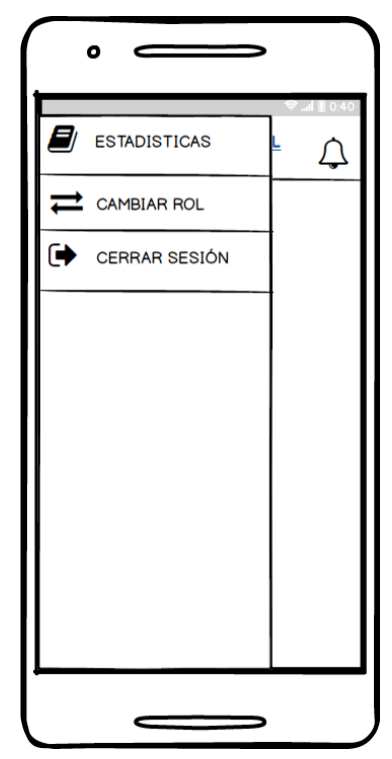

Figura 6.

Menú de principales funciones de la directora de escuela.

Las interfaces funcionales del menú del profesor, se aprecia en la figura 7.

Asistencias Hoy: Le permite gestionar o consultar las asistencias del día de fecha actual de las clases de sus cursos.

Asistencias: Le permite gestionar o consultar las asistencias de todas las clases de sus cursos.

Notas: Le permite gestionar o consultar las notas de los alumnos de sus cursos.

Preguntas y Respuestas: Le permite registrar o consultar preguntas y respuestas con los alumnos.

Anuncios: Le permite registrar o consultar anuncios de sus cursos.

Fórmula: Le permite gestionar o consultar fórmulas del cálculo de las notas de sus cursos.

Estadísticas: Le permite generar estadísticas sobre las asistencias, notas y encuestas de alumnos y profesores.

Cambiar Rol: Le permite cambiar de rol si tuviera más de uno.

Cerrar Sesión: Le permite cerrar la sesión

Las interfaces funcionales del menú del alumno, se aprecia en la figura 8.

Asistencias Hoy: Le permite consultar las asistencias del día de fecha actual de las clases de sus cursos.

Asistencias: Le permite consultar las asistencias de todas las clases de sus cursos.

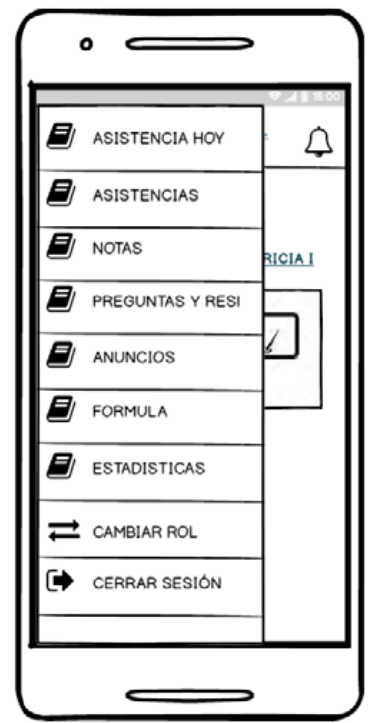

Figura 7.

Menú de principales funciones del profesor.

Notas: Le permite consultar las notas de sus cursos.

Preguntas y Respuestas: Le permite registrar o consultar preguntas y respuestas con los profesores y/o alumnos.

Anuncios: Le permite consultar los anuncios del profesor sobre sus cursos.

Fórmula: Le permite consultar fórmulas del cálculo de las notas de sus cursos.

Cambiar Rol: Le permite cambiar de rol si tuviera más de uno.

Cerrar Sesión: Le permite cerrar la sesión

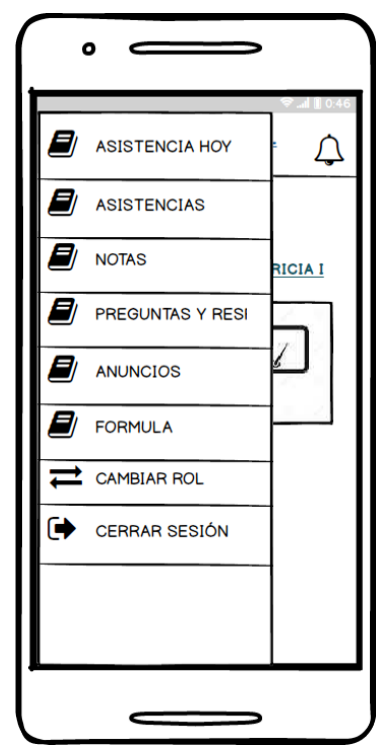

Figura 8.

Menú de principales funciones del alumno. 
Una de las funcionalidades más importantes del sistema se visualiza en la figura 9. Permitirá realizar el control general y específico de asistencias de alumnos y profesores por la directora de escuela. Los filtros que se presentan para lograr este fin son el curso, grupo (todos o uno específico), alumno (todos o uno específico), profesores (todos o uno específico), luego de haber escogido los criterios de filtros deseados se muestran estadísticas (que permitirán interpretar mejor la información) como avance de clases (porcentaje de clases avanzadas comparado con el total de clases) y dos gráficos por el momento de asistencias: el primero de profesores y el segundo de alumnos.

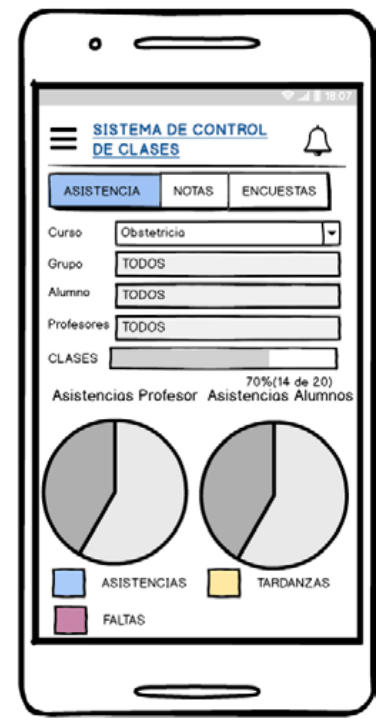

Figura 9.

Reporte/Consulta para control de asistencias de alumnos y profesores.

Fuente: Elaboración propia (2019).

\section{Conclusiones}

Se ha presentado una propuesta de diseño de un sistema móvil de control académico basado en microservicios y desarrollado con la metodología Scrum para la Escuela Profesional de Obstetricia (EPO) de la Universidad Nacional Mayor de San Marcos, en la se muestran las funcionalidades que debería tener el producto cuando se implemente, satisfaciendo las necesidades de información e integración. La arquitectura de microservicios presenta independencia, acoplamiento, cohesión, flexibilidad, despliegue y descubrimiento de servi- cios, por otro lado, la metodología Scrum permite la participación activa de los usuarios y mejor control para el logro de los entregables, así como una mejor gestión y organización del proyecto, facilitando el desarrollo y sobre todo permitir culminar el proyecto en los plazos establecidos.

Este artículo describe la parte de planificación, análisis y diseño del avance de una tesis de Ingeniería que aún está por concluirse, para su implementación previamente habría que probar los despliegues, así como una mejor distribución de los microservicios alojados en la base de datos, según tuning a realizar podría replantearse y refinarse la arquitectura presentada en la figura 5 .

El trabajo futuro a realizar sería continuar con las fases de desarrollo e implementación, la cual permitirá brindar una herramienta a la EPO y similares; facilitando la gestión académica y cumplir con los estándares planteados y exigidos por SINEACE.

\section{REFERENCIAS BIBLIOGRÁFICAS}

Abernethy, M.A. \& Chua, W.F. (1996). Un estudio de campo del "rediseńo" del sistema de control: el impacto de los procesos institucionales en la elección estratégica. Contemporary Accounting Research, 13 (2), 569-606. https://doi. org/10.1111/j.1911-3846.1996.tb00515.x

Arroyo, M.N. (2009) Influencia de la gestión pedagógica en el uso de las tecnologías de la información y comunicación en la Institución Educativa Darío Arrús de Bellavista, Callao. (Tesis inédita de Maestría). Universidad Nacional "Enrique Guzmán y Valle. Lima.

Beuren, I.M. \& Teixeira, SA (2014). Evaluación de sistemas de control de gestión en una institución de educación superior con gestión y control del desempeño https://doi. org/10.4301/S1807-17752014000100010

Britto, J.A. (2016). Comparación de metodologías ágiles y procesos de desarrollo de software mediante un instrumento basado en CMMI. Scientia et technica. 21 (2), 150-155. https://doi.org/10.22517/23447214.9249

Cerny, T., Donahoo, M. \& Pechanec, J. (2017). Desambiguación y comparación de SOA, microservicios y sistemas autónomos. Actas del RACS ' 17 de la Conferencia Internacional sobre Investigación en Sistemas Adaptativos y Convergentes (pp. 228-235). NY, EE. UU: Asociación de Maquinaria Informática. https://doi.org/10.1145/3129676.3129682 
Propuesta de arquitectura de microservicios, metodología Scrum para una aplicación móvil de control académico: Caso Escuela Profesional de Obstetricia de la Universidad Nacional Mayor de San Marcos

Che, Z. \& Rapiah, M. (2013). El efecto del sistema de control de gestión en el sistema de medición de desempeño en Small Medium Hotel en Malasia Revista Internacional de Comercio, Economía y Finanzas, 4 (4), 202-208. https:// doi.org/10.7763/IJTEF.2013.V4.286

COMEXPERU. (2011) Educación Superior: un diagnóstico. Semanario COMEXPERU (636), 5-6. Recuperado de http://www.comexperu.org.pe/media/files/semanario/SEMANARIO\%20COMEXPERU\%20636.PDF

Eisenman, B. (2016). Learning React Native (Primera edición). (M. Foley, Ed.) Estados Unidos de América: O’Reilly Media Inc.

Erl, T. (2016). SOA: Principios del diseño del servicio. NJ, EE. UU.: Prentice Hall.

Fernández, J.M. \& Cadelli, S. (2014). Convivencia de metodologías: Scrum y Rup en un proyecto de gran escala (Tesina de Licenciatura). Universidad Nacional La Plata, Argentina. Recuperado de http://hdl.handle.net/10915/47082

Ferreira, A. \& Otley, D. (2006). El diseño y uso de sistemas de gestión del desempeño: un marco extendido para el análisis. Investigación de contabilidad de gestión, 20 (4), 263282. https://doi.org/10.1016/j.mar.2009.07.003

Fowler, M. \& Lewis, J. (s.f). Guía de recursos de microservicios. Recuperado de https://martinfowler.com/microservices/

Haimann, T. (2005). En Dirección y gerencia: planificación, coordinación y control de las actividades de la empresa.

Horngren, C.T., Datar, S.M. \& Foster, G. (2000). Contabilidad de costos. Río de Janeiro: LTC.

Krafzig, D., Banke, K. \& Slama, D. (2004). Enterprise SOA: mejores prácticas de arquitectura orientada a servicios. Prentice Hall.

Moromi, NH (2002). La Influencia de la ejecución curricular y el uso de medios y materiales en el rendimiento académico de los estudiantes de la Facultad de Odontología de la Universidad Nacional Mayor de San Marcos (Tesis Postgrado). Universidad Nacional Mayor de San Marcos, Escuela de Postgrado - Unidad de Posgrado de la Facultad de Educación. Lima: Universidad Nacional Mayor de San Marcos. Recuperado de http://sisbib.unmsm.edu.pe/BibVirtual/Tesis/Human/Moromi_N_H/Moromi_N_H.htm

Newman, S. (2015). Construcción de microservicios. (ML MacDonald, Ed.) O'Reilly.

Pérez-Herrera, M. (2015). Arquitecturas basadas en microservicios. Madrid, España: UPM.

Quacquarelli, S. (2011). QS University Rankings: Latin America 2011. Recuperado el de https://content.qs.com/ supplement2011/Latin_American_supplement.pdf.

Quacquarelli, S. (2019). QS University Rankings: Latin America 2019. Quacquarelli Symonds: https://www.topuniversities.com/university-rankings/latin-american-university-rankings/2019

Rodríguez, C. \& Herrera, L. (2009). Análisis correlacional-predictivo de la influencia de la asistencia a clase en el rendimiento académico universitario. Estudio de caso en una asignatura. Profesorado: Revista de curriculum y formación del profesorado, 13 (2), 1-13. Recuperado de http:// www.redalyc.org/articulo.oa?id=56711798017

RV, R. (2016). Spring Microservices. Packt Publishing.

Sánchez Enriquez, JA (2011). Control de obtención del rendimiento de recursos humanos. (Thomson Reuters, Ed.).

Sifuentes, L.A. (2018). Desempeño didáctico y académico del docente relacionado con la satisfacción de los estudiantes del programa de Complementación Pedagógica de la Universidad Nacional Mayor de San Marcos, 2013-II. Recuperado de http://cybertesis.unmsm.edu.pe/handle/cybertesis/3981

SINEACE (2016) Modelo de Acreditación para Programas de Estudios de Educación Superior Universitaria. Lima, Perú. Recuperado de https:/www.sineace.gob.pe/ wp-content/uploads/2016/03/Anexo-a-la-Resoluci\%C3\%B3n-N\%C2\%B0022-2016-CDAH-P.pdf

Tolentino, L.A. (2014). Desempeño didáctico y académico del docente relacionado con la satisfacción de los estudiantes del programa de Complementación Pedagógica de la Universidad Nacional Mayor de San Marcos, 2013-II (Tesis Postgrado). Universidad Nacional Mayor de San Marcos, Facultad de Educación. Lima-Perú: Universidad Nacional Mayor de San Marcos. Recuperado de http://cybertesis.unmsm.edu.pe/handle/cybertesis/3981

Yanada, G., Rivera, M. \& Castro, J. (2012). Educación Superior en el Perú: Retos para el Aseguramiento de la Calidad. Perú: SINEACE. Recuperado de http://repositorio.minedu. gob.pe/handle/123456789/937 